\title{
Cutaneous Metastasis due to Breast Cancer in a Patient with Primary Biliary Cirrhosis: A Case Report
}

\author{
Sailaja Kamaraju Jill Depke Janice Povletich Adam Currey \\ Elizabeth Weil \\ Department of Internal Medicine, Department of Clinical Nursing, Department of \\ Radiation Oncology, and Department of Pharmacy, Froedtert/Medical College of \\ Wisconsin, Milwaukee, WI, USA
}

\section{Keywords}

HER-2-positive breast cancer - Cutaneous metastasis · Primary biliary cirrhosis .

Nab-paclitaxel

\begin{abstract}
Background: Breast cancer is the most common solid tumor to cause cutaneous metastases. These are incurable and the treatment goal is geared toward local control with surgical excision, radiation, and chemotherapy. However, treatment can be challenging in subjects with end-stage liver disease and a multidisciplinary approach is warranted. Case Report: In this case report, we present a 61-year-old female with primary biliary cirrhosis and human epidermal growth factor-2 (HER-2)-positive breast cancer, who subsequently developed cutaneous metastases. We briefly describe the treatment challenges due to underlying end-stage liver disease, and an exceptional response to trastuzumab and nab-paclitaxel. Conclusion: A multidisciplinary approach to local control and attenuated doses of nab-paclitaxel and trastuzumab suggest a durable response to HER-2-positive breast cancer with cutaneous metastasis. Subjects with end-stage liver disease pose unique challenges and toxicities, warranting additional research and drug development for less hepatotoxic antineoplastic agents.
\end{abstract}

(C) 2016 The Author(s)

Published by S. Karger AG, Basel 


\section{Case Reports in Oncology}

\section{Introduction}

Breast cancer is the most common solid malignancy to metastasize to skin [1-3]. The overall incidence of cutaneous metastasis is reported to range from 0.7 to $20 \%$ [3-5]. Median overall survival varied among the previous reports, ranging from 13.8 to 36 months [6]. While pigmented and nodular lesions are more frequent in melanoma, cutaneous metastases from breast cancer can present as solitary or multiple nodules (ulcerated, bullous) or as inflammatory, cicatricial lesions [3]. The underlying mechanism of skin involvement is unclear, but lymphangitic spread is considered to be the primary pathway to the development of cutaneous metastasis [7]. Skin lesions mimicking infections such as cellulitis can be misleading, and it is important that clinicians differentiate these for appropriate investigations and therapy. Other differential diagnoses can be sebaceous cyst, adnexal tumor, neurofibroma, and lipoma.

Primary biliary cirrhosis (PBC) is a suppurative destructive intrahepatic cholangitis, ultimately leading to cirrhosis of the liver. It is a heritable condition with familial prevalence, and the age-adjusted incidence was reported to be 4.5 (95\% confidence interval 3.1-5.9) for women and 0.7 (95\% confidence interval $0.1-1.3$ ) for men [8]. The exact mechanism of cirrhosis is unknown, but autoimmune mechanisms have been implicated, with increase in the risk of hepatocellular carcinoma as well as other cancer risk [9]. The risk of breast cancer was shown to be higher in some studies, whereas others showed no excess risk [10-14]. The overall prognosis of PBC is poor, with liver failure being the main cause of death. Patients are prone to liver failure-related complications such as esophageal varices, splenomegaly leading to cytopenias, and hepatocellular carcinoma [15].

\section{Case Report}

A 61-year-old Caucasian female with a prior diagnosis of PBC was diagnosed with T3N1M0 stage III, grade 2 left breast invasive ductal carcinoma in October 2011. The tumor was $7 \times 4 \mathrm{~cm}$, and a palpable left axillary lymph node was noted on examination. The tumor was positive for estrogen receptor, progesterone receptor, and HER-2 oncogene overexpression (fluorescence in situ hybridization ratio 9.91). Interestingly, her twin sister also had a diagnosis of PBC; other family history included lymphoma in maternal cousin and colon cancer in maternal uncle. Her case was presented at our institution's tumor board and neoadjuvant chemotherapy was recommended.

Due to the patient's underlying PBC, she had multiple baseline abnormalities of liver function tests, alkaline phosphatase [two times the upper limit of normal (ULN)] and grade 2 cytopenia from splenomegaly. Therefore, anthracycline-based regimens were avoided due to a concern for decreased clearance of doxorubicin leading to increased cardiotoxicity, as the patient would require trastuzumab as well. Neoadjuvant chemotherapy with 6 cycles of docetaxel, carboplatin, and trastuzumab (TCH) was given followed by bilateral skin-sparing total mastectomy and left axillary lymph node dissection with immediate reconstruction. Docetaxel was initiated with a $25 \%$ dose reduction and required a subsequent $25 \%$ dose reduction on carboplatin due to cytopenia. Postoperatively, pathology demonstrated a decrease in the tumor mass from $7 \times 4$ to $2.5 \times 2 \mathrm{~cm}$, but 4 of 20 axillary lymph nodes were 


\section{Case Reports in Oncology}

Kamaraju et al.: Cutaneous Metastasis due to Breast Cancer in a Patient with Primary Biliary Cirrhosis: A Case Report

positive for invasive cancer with extracapsular extension (T2N2a). Adjuvant radiation therapy was given to the chest wall and axilla $(5,000 \mathrm{~Gy})$. She then completed trastuzumab maintenance for a total of 52 weeks in October 2012 and started adjuvant endocrine therapy with anastrozole.

\section{Chest Wall (Cutaneous) Recurrence}

In February 2013, 3 months after the completion of trastuzumab maintenance therapy, our patient presented with skin nodules, and the clinical examination was remarkable for 2 erythematous left upper chest wall nodules measuring $0.5 \mathrm{~cm}$. Restaging positron-emission tomography-computed tomography demonstrated an increased activity in the anterior mediastinum and subcutaneous tissue along with medial left breast reconstruction area concerning for tumor recurrence. There was no evidence of distant/metastatic disease except for underlying cirrhosis of liver, portal hypertension, and splenomegaly. Punch biopsies of the skin nodules revealed invasive adenocarcinoma significant for a small area of lymphovascular invasion. Immunohistochemical stains for AE1-AE3 were positive, highlighting tumor cells, and CD31 was positive, highlighting the lymphovascular invasion. Cutaneous metastases were estrogen receptor negative, progesterone receptor negative and HER-2 positive. She required a bone marrow biopsy due to worsening of cytopenia, which revealed no evidence of breast cancer or other hematological malignancies.

The patient's unique presentation and complex comorbidities were discussed at the multidisciplinary tumor board. Based on the team's consensus, the patient was started on docetaxel, pertuzumab, and trastuzumab for 6 cycles, followed by pertuzumab and trastuzumab as maintenance therapy. Docetaxel was re-initiated at a $25 \%$ dose reduction $(65 \mathrm{mg} /$ $\mathrm{m}^{2}$ ). The patient's bilirubin was within normal limits; however, the patient's alkaline phosphatase was 1.6 times ULN, warranting a dose reduction. Repeat imaging was negative for systemic disease, except for the primary recurrence of cutaneous metastases. Unfortunately, she experienced a second recurrence of cutaneous metastasis while she was on anti-HER-2 therapy, for which she underwent surgical excision followed by radiation therapy $(6,000$ Gy). Our patient experienced multiple cutaneous recurrences over the period of the next 24 months. Due to abnormalities in liver function tests (bilirubin was over 2.5 times ULN), multiple anti-HER-2-based regimens were contraindicated, including trastuzumab emtansine (TDM1). Treatment with capecitabine, along with trastuzumab, was therefore initiated. The capecitabine dose was reduced by $25 \%$ based on the elevated bilirubin and anticipated patient tolerance, which was subsequently discontinued due to side effects and disease progression. Therapy was then changed to vinorelbine, along with trastuzumab. Vinorelbine was given with a $60 \%$ dose reduction $\left(12.5 \mathrm{mg} / \mathrm{m}^{2}\right)$ due to its extensive hepatic metabolism by cytochrome $\mathrm{P} 450$ enzymes. The patient's disease had progressed after 2 cycles of vinorelbine and it was discontinued.

The patient then developed a left breast implant infection and recurrent metastasis around the port and skin area, requiring removal of bilateral tissue expanders, full thickness excision of necrotic skin on the right chest wall, and antibiotic therapy. Right abdominal fasciocutaneous flap histology was negative for cancer. The patient's bilirubin was 2-2.5 times the ULN; subsequently, nab-paclitaxel at a $50 \%$ dose reduction was initiated every 3 weeks for 5 cycles along with trastuzumab, which resulted in complete resolution of the cutaneous metastasis. Our patient requested a drug holiday and decided to hold off chemo- 


\section{Case Reports in Oncology}

Kamaraju et al.: Cutaneous Metastasis due to Breast Cancer in a Patient with Primary Biliary Cirrhosis: A Case Report

therapy temporarily due to severe fatigue but agreed to continue trastuzumab every 3 weeks $(6 \mathrm{mg} / \mathrm{kg})$. One year later, she reported 2 small axillary nodules on the left upper chest wall and axilla concerning for recurrence. Unfortunately, her bilirubin was markedly elevated at 5 times ULN $(6.0 \mathrm{mg} / \mathrm{dL})$, limiting her ability to receive nab-paclitaxel.

\section{Discussion}

Cutaneous metastases from breast cancer are incurable and treatment is primarily geared toward local control with a multidisciplinary approach. While surgical resection and radiation therapy offer local control, treatment with antineoplastic therapy controls systemic disease. Our patient had an aggressive HER-2-positive breast cancer given her recurrence within 4 months of adjuvant trastuzumab maintenance therapy. The novel anti-HER-2 agents pertuzumab and ado-trastuzumab emtansine (TDM1) are very promising anti-HER-2 agents, which have shown a remarkable overall survival advantage in the advanced setting. Swain et al. [16] demonstrated a median overall survival of 56.5 months in subjects who received a 3-drug combination of pertuzumab, trastuzumab, and docetaxel (THP)-based therapy compared to 40.8 months for the latter 2 alone in metastatic breast cancer. Other trials incorporating lapatinib and TDM1 have shown promising results for HER-2-positive disease as well [17].

Despite the availability of several novel agents, our patient had impediments limiting the choices of antineoplastic agents due to: (1) hyperbilirubinemia restricting the therapy options, (2) cytopenia due to splenomegaly requiring dose reductions and treatment delays, and (3) peripheral edema due to third space fluid accumulation causing pharmacokinetic and pharmacodynamic challenges.

McDonald et al. [18] reported challenges in deciding appropriate medication dose adjustments for patients with liver disease based on the variable medication pharmacodynamics and kinetics. As seen below (Table 1), several antineoplastic agents are metabolized by the liver and contraindicated due to increased toxicity in the setting of hyperbilirubinemia. Additionally, several chemotherapeutic agents cause hepatotoxicity, requiring dose reductions and closer monitoring for toxicities (Table 1).

\section{Our Antineoplastic Approach/Management}

The current standard of care for recurrent HER-2-positive breast cancer is a combination of taxane (docetaxel or paclitaxel) and anti-HER-2 therapy as first-line regimen [19]. Ado-trastuzumab emtansine (TDM1) is the preferred second-line regimen based on a recent trial that demonstrated a median progression-free survival of 9.6 months with TDM1 compared to 6.4 months with lapatinib and capecitabine therapy $[16,17]$. Several other singleagent conventional chemotherapeutic agents in combination with anti-HER-2 therapy are offered as third-line agents [19].

Our multidisciplinary approach included pertuzumab, docetaxel, and trastuzumab with the recommendations of our institutional tumor board at first cutaneous recurrence. We optimized local control with surgical excision and locoregional radiation. Capecitabine (25\% dose reduction; $1,500 \mathrm{mg}$ b.i.d.) in combination with trastuzumab were administered as second-line, and vinorelbine ( $60 \%$ dose reduction; $12.5 \mathrm{mg} / \mathrm{m}^{2}$ ) with trastuzumab as third- 


\section{Case Reports in Oncology}

line therapy [20]. It is to be noted that our patient had several treatment delays and dose reductions due to the underlying $\mathrm{PBC}$, with 4-6 months of progression-free interval in between various chemotherapy regimens. Recently, she received trastuzumab $(6 \mathrm{mg} / \mathrm{kg}$ ) with nab-paclitaxel ( $50 \%$ dose reduction, $135 \mathrm{mg} / \mathrm{m}^{2}$ ) with near resolution of skin metastasis and enjoyed a progression-free survival of 11 months (Fig. 1 and 2 show the response prior to and after nab-paclitaxel).

Taxanes are microtubule inhibitors that are extensively metabolized in the liver and are contraindicated at certain thresholds. Patients with liver disease are at increased risk of toxicities due to reduced metabolism requiring dose reductions of multiple antineoplastic agents, including taxanes. Docetaxel is hepatically metabolized by the CYP3A4 isoenzyme. Mild to moderate liver impairment decreases clearance by $27 \%$ in vitro, resulting in a $38 \%$ increase in AUC based on the patients' liver function [21]. Nab-paclitaxel is extensively metabolized by the cytochrome P450 enzymes. Patients with moderate or severe hepatic impairment had 22-26\% decreased elimination and approximately $20 \%$ increase in the AUC compared with patients with normal hepatic function $[22,23]$. Due to reduced medication clearance in patients with hepatic impairment, they are at increased risk of toxicity, particularly from myelosuppression. Therefore, dose reductions are suggested for moderate to severe hepatic impairment and use is not suggested in patients with a bilirubin 5 times ULN [22]. However, in patients with multiple comorbidities, attenuated doses of nab-paclitaxel are feasible, as demonstrated in our patient, with a close supervision by the medical oncologist and hepatologist.

Our patient developed multiple wound infections, requiring surgical debridement and removal of infected breast implant. Despite these hurdles and the treatment delays, overall, she had an excellent response with nab-paclitaxel with resolution of skin metastases and thus far has no evidence of visceral or bone metastases.

\section{Conclusion}

Management of metastases in the presence of preexisting liver disease poses several clinical challenges as described in this case report. In our patient, a multidisciplinary approach and attenuated doses of nab-paclitaxel and trastuzumab provided a durable response. However, these patients can decompensate quickly due to sequelae of end-stage liver disease, and systemic therapy can be too toxic. Future research should focus on treatment options for local control and drug development for less hepatotoxic antineoplastic agents.

\section{Statement of Ethics}

A written permission was obtained from the patient for this case report.

\section{Disclosure Statement}

Thea authors declare that they have no disclosures. 


\section{Case Reports in Oncology}

\section{References}

1 Kalmykow B, Walker S: Cutaneous metastases in breast cancer. Clin J Oncol Nurs 2011;15:99-101.

-2 Nava G, et al: Metastatic cutaneous breast carcinoma: a case report and review of the literature. Can J Plast Surg 2009;17:25-27.

-3 Lookingbill DP, Spangler N, Helm KF: Cutaneous metastases in patients with metastatic carcinoma: a retrospective study of 4,020 patients. J Am Acad Dermatol 1993;29:228-236.

Spencer PS, Helm TN: Skin metastases in cancer patients. Cutis 1987;39:119-121.

Rosen T: Cutaneous metastases. Med Clin North Am 1980;64:885-900.

Schoenlaub P, et al: Survival after cutaneous metastasis: a study of 200 cases (in French). Ann Dermatol Venereol 2001;128:1310-1315.

Brodland DG, Zitelli JA: Mechanisms of metastasis. J Am Acad Dermatol 1992;27:1-8.

Kim WR, et al: Epidemiology and natural history of primary biliary cirrhosis in a U.S. community. Gastroenterology 2000;119:1631-1636.

Kouroumalis E, Notas G: Primary biliary cirrhosis: from bench to bedside. World J Gastrointest Pharmacol Ther 2015;6:32-58.

-10 Boonstra K, et al: Increased cancer risk in a large population-based cohort of patients with primary biliary cirrhosis: follow-up for up to 36 years. Hepatol Int 2014;8:266-274.

11 Jones DE, et al: Hepatocellular carcinoma in primary biliary cirrhosis and its impact on outcomes. Hepatology 1997;26:1138-1142.

$>12$ Wolke AM, et al: Malignancy in primary biliary cirrhosis. Am J Med 1984;76:1075-1078. Goudie BM, et al: Breast cancer in women with primary biliary cirrhosis. Br Med J (Clin Res Ed) 1985;291:1597-1598.

14 Loof L, et al: Cancer risk in primary biliary cirrhosis: a population-based study from Sweden. Hepatology 1994;20(1 Pt 1):101-104. Abe M, Onji M: Natural history of primary biliary cirrhosis. Hepatol Res 2008;38:639-645. Swain SM, et al: Pertuzumab, trastuzumab, and docetaxel in HER2-positive metastatic breast cancer. N Engl J Med 2015;372:724-734.

17 Verma S, et al: Trastuzumab emtansine for HER2-positive advanced breast cancer. N Engl J Med 2012;367:1783-1791.

18 McDonald GB, Frieze D: A problem-oriented approach to liver disease in oncology patients. Gut 2008;57:987-1003.

19 Carlson RW, et al: Metastatic breast cancer, version 1.2012: featured updates to the NCCN guidelines. J Natl Compr Canc Netw 2012;10:821-829.

-20 Burstein HJ, et al: Clinical activity of trastuzumab and vinorelbine in women with HER2-overexpressing metastatic breast cancer. J Clin Oncol 2001;19:2722-2730.

-21 Eckmann K, et al: Pilot study to assess toxicity and pharmacokinetics of docetaxel in patients with metastatic breast cancer and impaired liver function secondary to hepatic metastases. J Oncol Pharm Pract 2014;20:120-129.

22 Chen N, et al: Pharmacokinetics and pharmacodynamics of nab-paclitaxel in patients with solid tumors: disposition kinetics and pharmacology distinct from solvent-based paclitaxel. J Clin Pharmacol 2014;54:1097-1107.

-23 Biakhov MY, et al: Nab-paclitaxel in patients with advanced solid tumors and hepatic dysfunction: a pilot study. Expert Opin Drug Saf 2010;9:515-523. 


\section{Case Reports in Oncology}

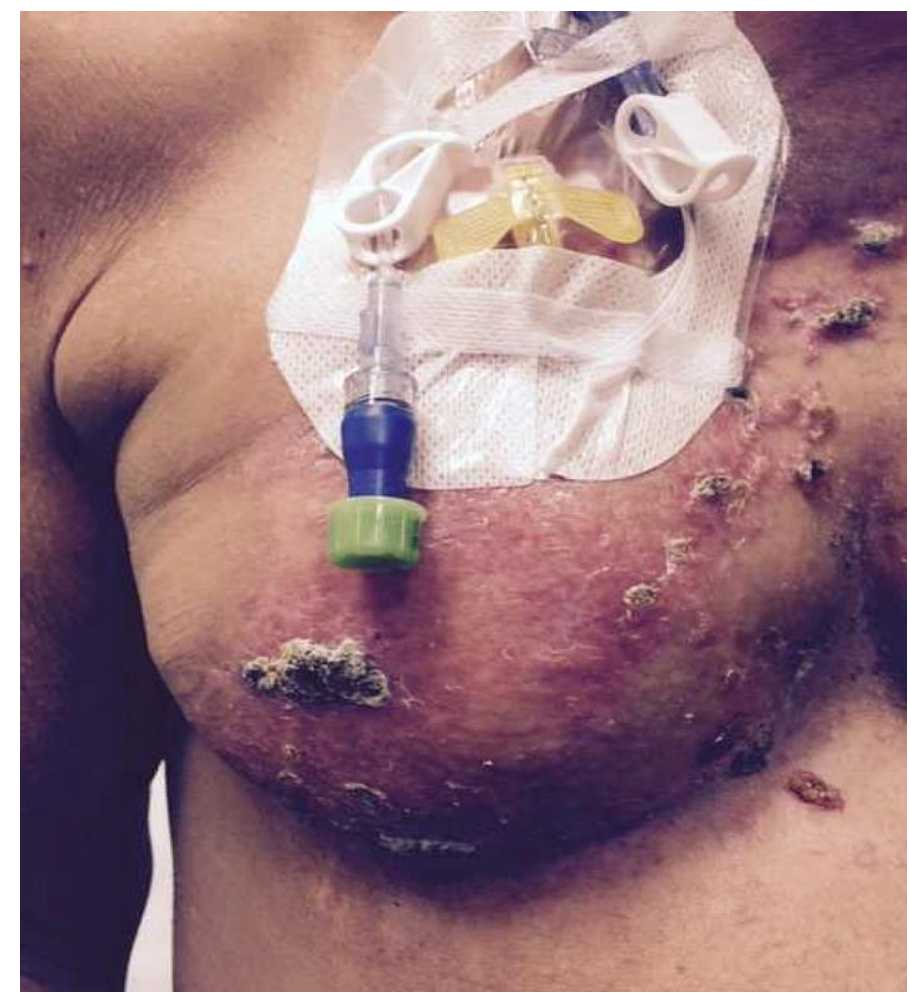

Fig. 1. Metastatic lesions on the breast, around the port and chest wall, before nab-paclitaxel.

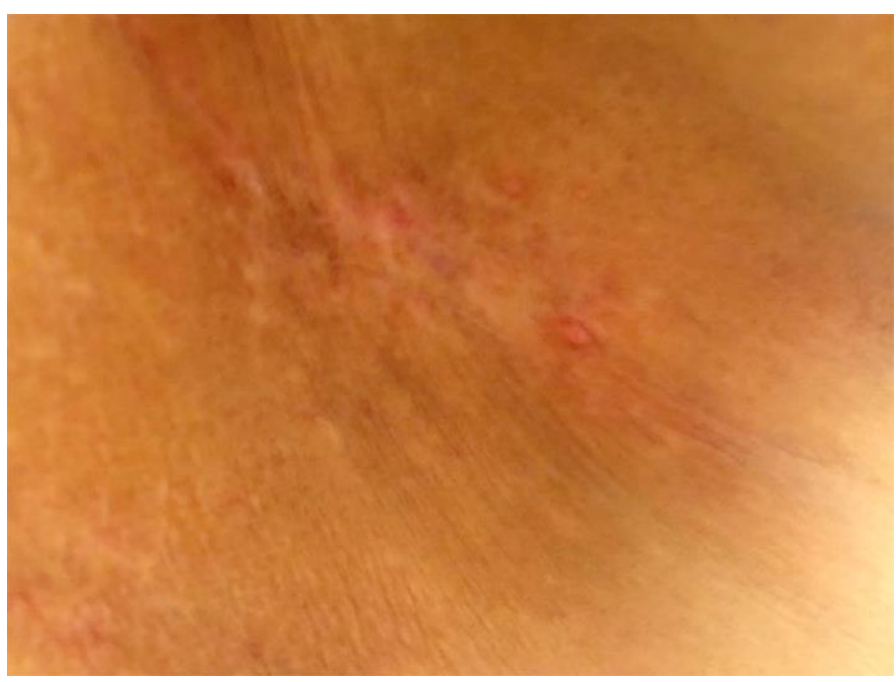

Fig. 2. Near resolution of chest wall lesions after nab-paclitaxel therapy. 


\section{Case Reports in Oncology}

Kamaraju et al.: Cutaneous Metastasis due to Breast Cancer in a Patient with Primary Biliary Cirrhosis: A Case Report

Table 1. Hepatotoxicities due to chemotherapeutic agents

Toxicities due to chemotherapeutic agents

Hepatocellular necrosis

Cyclophosphamide

Gemcitabine

Methotrexate

Cholestatic injury

Tamoxifen

Sinusoidal or vascular liver injury

Carboplatin

Cyclophosphamide

Doxorubicin

Methotrexate

Tamoxifen

\begin{tabular}{ll}
\hline Steatosis & Methotrexate \\
& Tamoxifen \\
\hline Hyperbilirubinemia & Taxanes \\
& Ixabepilone \\
& Eribulin
\end{tabular}

Other anti-HER-2 agent-related hepatotoxicity

No significant hepatotoxicity

Trastuzumab

No significant hepatotoxicity

Pertuzumab

Immune-mediated liver injury

Lapatinib

Intracellular damage of hepatocytes (dose dependent)

Ado-trastuzumab emtansine (TDM1) 